\section{Berufsverband der Deutschen Urologen e.V.} Urologe 2015 · 54:756

DOI 10.1007/s00120-015-3832-1

๑) Springer-Verlag Berlin Heidelberg 2015
Redaktion

W. Bühmann,Wenningstedt/Sylt

\title{
$\#+4$ URO-Telegramm
}

\section{Abrechnungsbetrug ist kein Massenphänomen}

Ermittler der DAK-Gesundheit gehen derzeit knapp 1.700 Hinweisen auf Abrechnungsbetrug nach. Im vergangenen Jahr habe die Ermittlungsgruppe deutschlandweit 1.000 Fälle abgeschlossen und 1,3 Millionen Euro an unrechtmäßig gezahlten Geldern zurückgeholt, teilte die Krankenkasse am Mittwoch in Hamburg mit. Heilmittel wie zum Beispiel Physiotherapie, Krankengymnastik und Massagen, bildeten demnach mit 41 Prozent der Fälle den Schwerpunkt der Ermittlungen. Es folgten der Bereich Pflege mit 17 und Ärzte mit 12 Prozent.

Betrogen werde mit gefälschten Rezepten, erfundenen Behandlungen und manipulierten Rechnungen. "Soweit es möglich ist, gehen wir konsequent gegen die Betrüger vor und holen uns das Geld zurück, weil wir dies unseren Versicherten schuldig sind", sagte der Leiter der zehnköpfigen DAK-Ermittlungsgruppe, Volker zur Heide.

Abrechnungsbetrug sei aber kein Massenphänomen. Die Kasse unterstützt die Pläne der Bundesregierung für ein Gesetz gegen Korruption im Gesundheitswesen. Von den erfolgreichen Rückforderungen in Höhe von 1,3 Millionen Euro im vergangenen Jahr entfielen 392000 auf den Bereich Heilmittel, 247000 Euro waren für Arznei- und Verbandmittel deklariert worden. 211000 Euro flossen aus dem Pflegebereich an die DAK zurück, 120000 Euro holte sich die Kasse bei Ärzten. Der Rest der Geldrückflüsse betraf Hilfsmittel (96 000), Zahnärzte (65 000) und Krankentransporte (35 000 Euro). ๑) dpa/aerzteblatt.de

Kommentar der Redaktion: Siehe da, alle Ärzte sind Verbrecher und betrügen systematisch - für 120.000 ,- bei $160 \mathrm{Mrd}$. Jahresetat in der GKV ... - wir brauchen dringend neue Gesetze, um diese Massenkriminalität zu geißeln! Unser Ansehen ist wirklich ungebrochen - wie sonst ist ein solches Maß an Sozialneid zu erklären?

\section{Statistisches Bundesamt: Gesundheitsausgaben steigen}

Die Ausgaben für Gesundheit steigen stärker als die Wirtschaftsleistung Deutschlands.

Das berichtete das Statistische Bundesamt am Dienstag in Wiesbaden. Jeder zehnte Euro fließt in diesen Sektor. 2013 waren das 3910 Euro pro Bürger.

Insgesamt betrugen die Gesamtgesundheitsausgaben 314,9 Milliarden Euro, vier Prozent mehr als 2012. Der Anteil am Bruttoinlandsprodukt lag bei 11,2 Prozent - Tendenz steigend: 2012 waren es 11, o Prozent, 2011 10,9 Prozent.

„Der leichte Anstieg gegenüber dem Vorjahr ist durch den stärkeren Anstieg der Gesundheitsausgaben im Vergleich zur Wirtschaftsleistung zu erklären", berichtete Destatis. Mehr als die Hälfte der Ausgaben zahlte die gesetzliche Krankenversicherung (181,5 Milliarden Euro, plus 5,3 Prozent). Privatpersonen übernahmen knapp 14 Prozent $(42,9$ Milliarden Euro, minus 0,6 Prozent): dass sie entlastet wurden, lag am Wegfall der Praxisgebühr.

Die privaten Krankenkassen trugen knapp zehn Prozent der Kosten (28,9 Milliarden Euro, plus 3,3 Prozent). Die Hälfte der Ausgaben entfiel auf Güter und Dienstleistungen im ambulanten Bereich (155,5 Milliarden Euro, 4,1 Prozent mehr).

Den stärksten Anstieg gab es bei ambulanten Pflegeeinrichtungen (12,3 Milliarden Euro, 9,0 Prozent mehr) und bei sonstigen medizinischen Praxen wie Physiotherapie (11,0 Milliarden Euro, plus 5,9 Prozent).

(dpa)

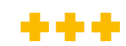

\section{Heer der Harninkontinen- ten wächst stetig - Neue Versorgungskonzepte vonnöten}

Rund $50 \%$ der 60 - bis 70-Jährigen beklagen Symptome der überaktiven Blase (ÜAB); $30-40 \%$ von diesen leiden unter einer Belastungs- und/ oder Dranginkontinenz. Diese Zahlen nannte Prof. KlausPeter Jünemann, Direktor der Klinik für Urologie und Kinderurologie, Campus Kiel, und scheidender Erster Vorsitzender der Deutschen Kontinenz Gesellschaft e.V. bei der Jahrestagung der Deutschen Kontinenz Gesellschaft in Frankfurt am Main. Mit zunehmendem Alter, vor allem aber bei einem Ortswechsel in ein Senioren- oder Pflegeheim steigen die Harninkontinenzraten dramatisch. Nicht selten erreichen sie bis zu 90\%. Gleichzeitig nimmt der Ärztemangel ungebremst zu. Angesichts der Zahlen aus einer aktuellen Hochrechnung des deutschen Krankenhausinstituts, nach der schon 2019 mehr als 37.000 Ärzte in Praxen und Krankenhäusern fehlen, sind neue Konzepte dringend gefragt, um die Versorgung harninkontinenter alter Menschen zu sichern, mahnte der Urologe.

Einen Lösungsansatz sieht die Deutsche Kontinenzgesellschaft in der Spezialausbildung von Krankenschwestern, wie sie in Österreich bereits stattfindet. Diese Fachkräfte mit Zusatzqualifikation müssten nicht nur die Kompetenz für einfache differenzialdiagnostische Schlüsse erwerben, sondern sie auch in therapeutische Konsequenzen umsetzen können. „Bleibt zu hoffen, dass die Politik in diesem Fall zuhört und die Rahmenbedingungen dafür schafft", schloss Jünemann.

Ute Ayazpoor; URO-NEWS 2015/7

\section{Kinderbeauftragter im Bundestag: Kinderarzt erfolgreich mit seiner Petition}

Die Zustimmung für das Vorhaben, einen Kinder- und Jugendbeauftragten des Deutschen Bundestages einzusetzen, ist groß. Mehr als 100.000 Kinder, Jugendliche und Erwachsene haben eine Petition von Manfred Gahr unterstützt, dem Generalsekretär der Deutschen Akademie für Kinder- und Jugendmedizin (DAKJ). Nun wird es voraussichtlich im Juni zu einer öffentlichen Anhörung im Petitionsausschuss über Gahrs Anliegen kommen.

„Erfreulich war, dass uns viele engagierte Bürgerinnen und Bürger, Eltern und Großeltern sowie auch Jugendliche selbst angeschrieben haben, die immer den neusten Stand der Petition wissen wollten und sich in ihrem Umfeld dafür eingesetzt haben", berichtete Gahr. Er erinnerte daran, dass auch zahlreiche Politiker in der Vergangenheit bereits einen Bundeskinderbeauftragten gefordert hatten. 